\title{
Acute cerebellitis requiring posterior fossa decompression after COVID-19 vaccination in an ocrelizumab-treated patient with multiple sclerosis
}

\author{
Gregor Brecl Jakob ${ }^{1}\left[\right.$ ] Lina Savšek ${ }^{2} \cdot$ Bernard Meglič $^{1}$
}

Received: 16 February 2022 / Accepted: 22 February 2022 / Published online: 28 February 2022

(c) Fondazione Società Italiana di Neurologia 2022

\section{Introduction}

Vaccination against COVID-19 is highly recommended for patients with multiple sclerosis (MS), especially those treated with anti-B-cell therapies [1]. The incidence of neurological complications of COVID-19 vaccines in the general population seems to be comparable with other vaccines (e.g., against influenza...) [2, 3]. However, little is known about the possible specific adverse events of COVID-19 vaccines in patients with autoimmune diseases such as MS and/ or in subjects using immune therapies.

We report a case of an ocrelizumab-treated female MS patient, who suffered a fulminant cerebellitis after the first dose of COVID-19 vaccine.

\section{Case report}

A 39-year-old female with a history of MS from the age of 22 was initially treated for MS with IFN-1b from 2005 to 2011. Despite treatment, she suffered several relapses. In 2011, natalizumab therapy was recommended; however, the patient stopped the treatment until April 2013, when she gave birth to a healthy daughter. After giving birth, she suffered a psychotic episode, requiring psychiatric in-patient care, leading to full recovery. In February 2014, natalizumab treatment was commenced. She received 65 infusions, of

The adverse event reported here has been reported to the National Institute of Public Health (NIJZ) (Slovenia).

Gregor Brecl Jakob

gregor.brecljakob@kclj.si

1 Department of Neurology, University Medical Centre Ljubljana, Ljubljana, Slovenia

2 Department of Neurology, Celje General Hospital, Celje, Slovenia which the last 15 were administered at the extended dosing interval of 6 weeks. In November 2019, the patient was switched to ocrelizumab due to high anti-JCV antibody index (3.54), followed by two infusions, last one being administered in February 2021. During this time, her MS remained stable. She received her first dose of COVID-19 vaccine (BNT162b2 vaccine) on June 10th, 2021.

The patient presented on June 27th, 2021, with a 2-day history of fatigue and weakness followed by fever $\left(39-40^{\circ} \mathrm{C}\right)$. At that time, she was treated with lamotrigine, venlafaxine, and depot-olanzapine. Neurological examination revealed no new neurological deficits beside her previously known MS disability (EDSS 3.0). Urinalysis and chest X-ray were normal and SARS-CoV-2 infection was excluded.

In the following 2 days, her consciousness deteriorated to sopor. Brain CT scan showed no abnormalities. Blood tests were insignificant (mild leukocytosis $\left(13.2 \times 10^{9}\right.$ cells/ $\mathrm{mL}$ ), mild CRP elevation ( $26.4 \mathrm{mmol} / \mathrm{L})$, and normal procalcitonin levels).

She was transferred to the intensive care unit (ICU). CSF examination, performed on June 30th, showed lymphocytic pleocytosis (Leu 22, Lym 21, 20 RBC due to mildly traumatic lumbar puncture, no xantochromia), mildly elevated proteins $(0.64 \mathrm{~g} / \mathrm{L})$, and normal glucose level. In addition to the previously known brain and cervical spine MS lesions, slight T2/FLAIR hyperintensities of the cerebellar cortex were observed. Extensive workup to exclude infectious diseases was performed and no pathogen was identified. Empirical treatment with ceftriaxone, ampicillin, and acyclovir was started. Despite this, her consciousness deteriorated to coma, requiring artificial ventilation. Follow-up CT scan showed diffuse cytotoxic oedema of cerebellum with compression of the structures of the posterior fossa and hydrocephalus mandating external ventricular drainage. Short-term dexamethasone treatment was instituted. On July 4th, the brain MRI showed 
diffuse cytotoxic oedema of cerebellar cortex with restriction of diffusion and leptomeningeal contrast enhancement of cerebellar foliae. Brainstem and cerebellar white matter were not affected (Fig. 1A). Treatment with corticosteroids was started; however, on July 6th posterior fossa decompression was necessary due to ascendant herniation. Extensive and repeated microbiological and immunological tests (CSF culture; CSF eubacterial PCR; CSF PCR for neurotropic viruses, hepatitis E virus Listeria, Mycoplasma and Chlamydia; CSF and serum paraneoplastic antibodies and antibodies against surface neuronal antigens; Lyme disease serology; Central European Tick-Borne Encephalitis serology; nasopharyngeal swab with respiratory viruses PCR panel) revealed no possible etiological pathogen.

During 30 days of ICU treatment, she suffered several infections and developed critical illness myopathy. She remained severely disabled (EDSS 9.5) with tetraplegia, marked postural instability, and severe dysphagia, requiring a gastrostomy. Follow-up MRI showed marked cerebellar atrophy and numerous new MS lesions at various brain locations (Fig. 1B). Treatment with ocrelizumab was not continued, and to control the MS disease activity, she was switched to cladribine. After several aspiration pneumonias, the patient died 6 months after the beginning of disease.

\section{Discussion}

Acute cerebellitis is rare in adults. Most reports come from pediatric population, and it is considered to be either of infectious or postinfectious etiology. In adults, only a few reports exist. Recently, Van Samkar et al. report a similar case to ours: a 37-year-old female with diffuse cerebellitis, possibly related to a recent EBV and/or CMV infection. The patient recovered fully after a combination of ceftriaxone and acyclovir, combined with dexamethasone. In the review of 35 adult patients with acute cerebellitis, they found $34 \%$ of the cases to be of unknown etiology, while $11 \%$ were medication-induced, $9 \%$ paraneoplastic, and $6 \%$ para-infectious. Twenty-six percent of these cases evolved into hydrocephalus and mean CSF leukocyte count was 104 $/ \mathrm{ml}$ (range $0-797$ ). The patients fully recovered in $53 \%$ of the cases and in only $19 \%$ cerebellar atrophy was observed at follow-up [4].
Fig. 1 A Slight T2/FLAIR hyperintensities of the cerebellar cortex; B diffuse cytotoxic oedema of cerebellar cortex with restriction of diffusion (right image) sparing deep white matter and brainstem; $\mathbf{C}$ marked cerebellar atrophy
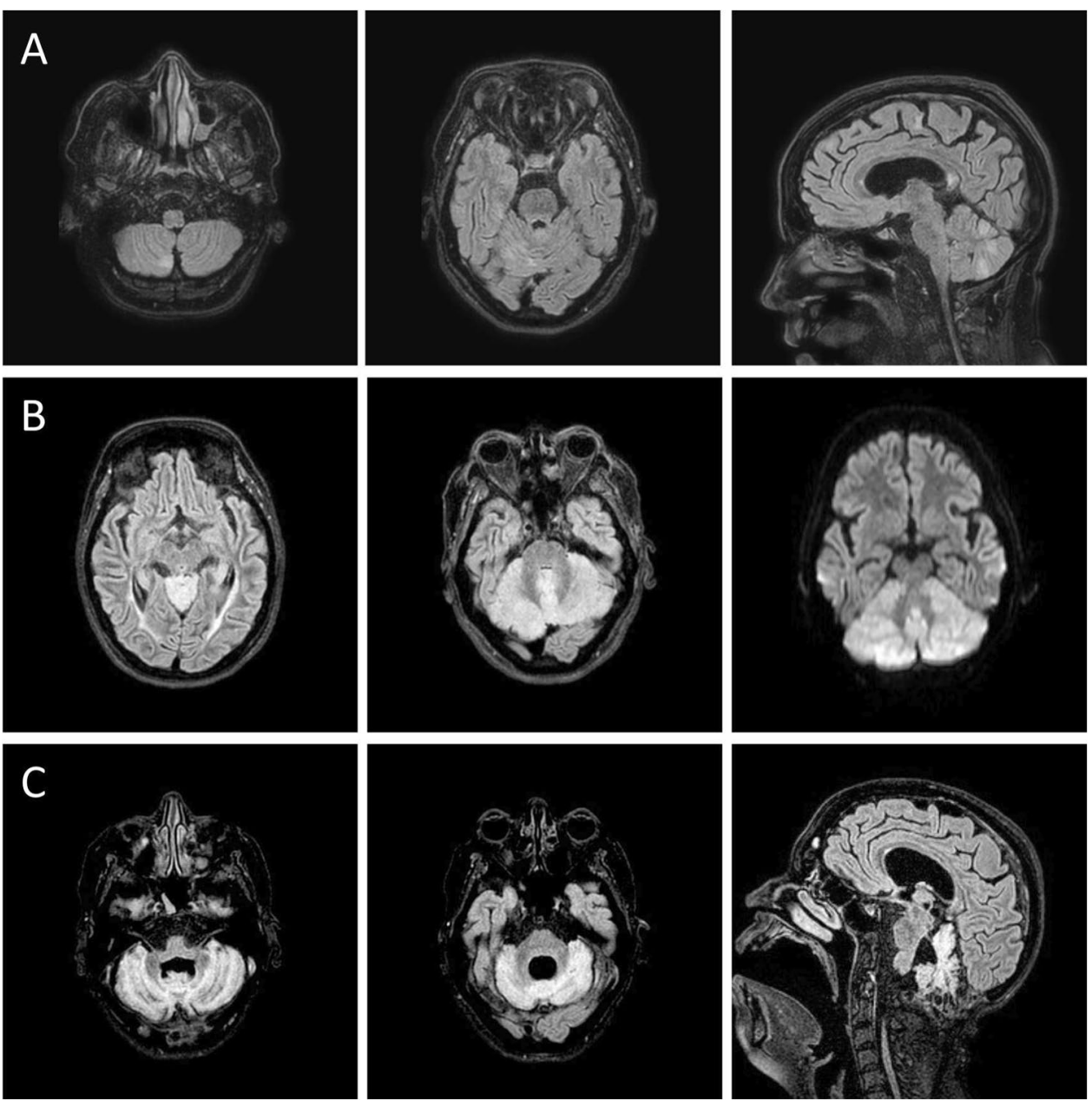
Our case differs from the reported cases in several aspects. It was a fulminant and treatment-resistant cerebellitis exhibiting diffuse, rather than focal (as in most published cases) cerebellar involvement, primarily limited to the cerebellar cortex. This raised a suspicion of toxic cerebellitis; however, no such agents were identified.

There are no reports of cerebellitis following COVID-19 vaccination. Additionally, we found no cases of acute cerebellitis as a possible adverse event of anti-CD20 treatment.

In our case, two factors should be considered. Firstly, the temporal relation of cerebellitis to COVID-19 vaccination suggests possible causality. On the other hand, the possible contribution of ocrelizumab treatment cannot be excluded. At the time of the cerebellitis, our patient's CD19/20 lymphocyte counts were still zero. This could cause some level of immune dysregulation since B-lymphocytes are known to exert a regulatory role on other immune cells, especially T-lymphocytes. Cases of immune dysregulations after anti-CD20 treatment have been previously described (e.g., exacerbation of psoriasis) [5]. The very uniform and diffuse cerebellitis with lymphocytic pleocytosis and unidentified pathogen indicates an immune-mediated process against an antigen, very specific for cerebellar cortex. There have been reports of acute cerebellitis related to SARS-CoV-2 infection [6] which could, in addition to our case, indicate an existence of an antigen (on the virus itself or on the vaccine-generated S-protein) triggering the immune-mediated cerebellitis.

In conclusion, the presented case suggests possible relationship between the COVID-19 vaccination and acute cerebellitis.

Author contribution Study concept and design: Brecl Jakob, Meglič. Acquisition of data: Brecl Jakob, Savšek, Meglič, analysis and interpretation of data: Brecl Jakob, Savšek, Meglič. Drafting of the manuscript: Brecl Jakob. Critical revision of the manuscript for important intellectual content: Brecl Jakob, Savšek, Meglič. Administrative, technical, and material support: Brecl Jakob.

\section{Declarations}

Ethical approval The patient gave the informed consent statement to publish her case.

Conflict of interest GBJ: participated as a clinical investigator and/or received consultation and/or speaker fees from: Biogen, Sanofi Genzyme, Merck, Novartis, Pliva/Teva, Roche, Lek, Astra-Zeneca.

LS: personal fees (speaker honoraria and travel grants) from Bayer, Biogen, Boehringer Ingelheim, Genzyme, Krka, Merck, Novartis, Pfizer, Roche, Sandoz, Sanofi and Teva.

BM: personal fees (speaker honoraria and travel grants) from Sandoz, Krka.

\section{References}

1. Woopen C, Schleussner K, Akgun K, Ziemssen T (2021) Approach to SARS-CoV-2 vaccination in patients with multiple sclerosis. Front Immunol 12:701752

2. Achiron A, Dolev M, Menascu S, Zohar DN, Dreyer-Alster S, Miron S, Shirbint E, Magalashvili D, Flechter S, Givon U, Guber D, Stern Y, Polliack M, Falb R, Gurevich M (2021) COVID-19 vaccination in patients with multiple sclerosis: what we have learnt by February 2021. Mult Scler 27(6):864-870

3. Patone M, Handunnetthi L, Saatci D, Pan J, Katikireddi SV, Razvi S, Hunt D, Mei XW, Dixon S, Zaccardi F, Khunti K, Watkinson P, Coupland CAC, Doidge J, Harrison DA, Ravanan R, Sheikh A, Robertson C, Hippisley-Cox J (2021) Neurological complications after first dose of COVID-19 vaccines and SARS-CoV-2 infection. Nat Med 27(12):2144-2153

4. Van Samkar A, Poulsen MNF, Bienfait HP, Van Leeuwen RB (2017) Acute cerebellitis in adults: a case report and review of the literature. BMC Res Notes 10(1):610

5. Darwin E, Romanelli P, Lev-Tov H (2018) Ocrelizumab-induced psoriasiform dermatitis in a patient with multiple sclerosis. Dermatol Online J 24(7)

6. Moreno-Escobar MC, Feizi P, Podury S, Tandon M, Munir B, Alvi M, Adcock A, Sriwastava S (2021) Acute cerebellitis following SARS-CoV-2 infection: a case report and review of the literature. J Med Virol 93(12):6818-6821

Publisher's note Springer Nature remains neutral with regard to jurisdictional claims in published maps and institutional affiliations. 